\title{
VON NEUMANN ALGEBRAS WHICH ARE SECOND DUAL SPACES
}

\author{
CHO-HO CHU
}

(Communicated by William J. Davis)

\begin{abstract}
A $\sigma$-finite von Neumann algebra is a second dual if and only if it
\end{abstract} is atomic.

Shtern [7] has shown that a $C^{*}$-algebra is a third dual if and only if it is the enveloping von Neumann algebra of a von Neumann algebra. $C^{*}$-algebras that are dual spaces are the von Neumann algebras. The remaining question appears to be: when is a $C^{*}$-algebra a second dual? This question is the same as asking when a von Neumann algebra is a second dual. Let $M$ be a von Neumann algebra and let $M=X^{* *}$ for some Banach space $X$. By the Krein-Milman Theorem, $M$ has a pure normal state and hence $M$ has a type I summand. Can $M$ have type II and type III summands as well? The answer is yes if, for instance, $X$ is the quotient of the algebra $B(H)$ of bounded operators on a separable Hilbert space $H$ by the compact operators $K(H)[1,6]$. The above observation seems to suggest, without some restriction, that the question may be too general to have a fruitful answer. If, however, we restrict the question to the class of $\sigma$-finite von Neumann algebras, then a simple answer emerges.

Theorem. $A$-finite von Neumann algebra $M$ is a second dual space if and only if $M$ is atomic, that is, if and only if $M$ is a direct sum of the $B(H)$ 's.

In the commutative case, the above is of course a well-known result of Pelczynski [4, Theorem 4] since a commutative $\sigma$-finite von Neumann algebra is just an $L_{\infty}(\mu)$ where $\mu$ is a $\sigma$-finite measure and in this case, Pelczynski has shown that $L_{1}(\mu)$ is (isomorphic to) a dual if and only if $\mu$ is purely atomic. Rosenthal [5, Corollary 2.2] has given a short proof of this result. Distel and Uhl [3, p. 83] gave a shorter proof using the Radon-Nikodym property and their approach works well in the noncommutative case.

Recall that the $\sigma$-finite von Neumann algebras are those that admit faithful normal states. Examples: (i) any von Neumann algebra acting on a separable Hilbert space; (ii) all von Neumann algebras appeared in quantum statistical mechanics and quantum field theory. A Banach space is called weakly compactly generated (WCG) if it is the (norm) closed linear span of one of its

Received by the editors February 1, 1990 and, in revised form, August 20, 1990.

1980 Mathematics Subject Classification (1985 Revision). Primary 46L10, 46B22. 
weakly compact subsets. Infinite-dimensional von Neumann algebras are not WCG since a WCG von Neumann algebra has the Radon-Nikodym property [3, p. 87] and must be finite dimensional.

Lemma. $A$ von Neumann algebra $M$ is $\sigma$-finite if and only if its predual $M_{*}$ is $W C G$.

Proof. Suppose $M$ has a faithful normal state $\varphi \in M_{*}$. By [8, Proposition 5.12], the set $K=\left\{\varphi\left(a_{0}\right): a \in M,\|a\| \leq 1\right\}$ is a relatively $\sigma\left(M_{*}, M\right)$-compact set in $M_{*}$. The linear span lin $K$ is (norm) dense in $M_{*}$. Otherwise, there exists nonzero $b$ in $M$ with $\|b\| \leq 1$ such that $\langle\psi, b\rangle=0$ for all $\psi \in \operatorname{lin} K$. If $b=u|b|$ is the polar decomposition, then $\langle\varphi,|b|\rangle=\left\langle\varphi\left(u^{*}.\right), b\right\rangle=0$ as $\varphi\left(u^{*}.\right) \in K$, contradicting the faithfulness of $\varphi$. So $M_{*}$ is WCG. Conversely, let $M_{*}$ be generated by a $\sigma\left(M_{*}, M\right)$-compact set $S$. By [8, Theorem 5.4], there is a normal state $\omega \in M_{*}$ with the property that for any $\varepsilon>0$, there exists $\delta>0$ such that $|\langle\psi, a\rangle|<\varepsilon$ for all $\psi \in S$ whenever $\|a\| \leq 1$ and $\left\langle\omega, a^{*} a+a a^{*}\right\rangle\left\langle\delta\right.$. Hence $\left\langle\omega, a^{*} a\right\rangle=0$ implies $\left\langle\psi,\left(a^{*} a\right)^{1 / 2}\right\rangle=0$ for all $\psi \in S$ and so $\left\langle\psi,\left(a^{*} a\right)^{1 / 2}\right\rangle=0$ for all $\psi \in M_{*}$, giving $a=0$. That is, $\omega$ is faithful.

Now, if a von Neumann algebra $M$ is $\sigma$-finite as well as a second dual, then its predual $M_{*}$ is WCG and a dual. So $M_{*}$ has the Radon-Nikodym property [3, p. 87]. By [2, Theorem 4], $M$ is atomic.

\section{REFERENCES}

1. J. Anderson and J. Bunce, A type $\mathrm{II}_{\infty}$ factor representation of the Calkin algebra, Amer. J. Math. 99 (1977), 515-521.

2. C.-H. Chu, A note on scattered $C^{*}$-algebras and the Radon-Nikodym property, J. London Math. Soc. 24 (1981), 533-536.

3. J. Diestel and J. J. Uhl, Vector measures, Math. Surveys, vol. 15, Amer. Math. Soc., Providence, R.I., 1977.

4. A. Pelczynski, On Banach spaces containing $L_{1}(\mu)$, Studia Math. 30 (1968), 231-246.

5. H. P. Rosenthal, On injective Banach spaces and the space $L^{\infty}(\mu)$ for finite measures $\mu$, Acta Math. 124 (1970), 205-248.

6. S. Sakai, A problem of Calkin, Amer. J. Math. 88 (1966), 935-941.

7. A. I. Shtern, Second dual of a von Neumann algebra, Funktsional. Anal. i Prilozhen. 16 (1982), 90.

8. M. Takesaki, Theory of operator algebras I, Springer-Verlag, Berlin, 1979.

Goldsmiths' College, University of London, London S.E.14, England 